\title{
Role of Ion-Ion Recombination for Alkali Chloride Cluster Formation in Liquid Secondary Ion Mass Spectrometry
}

\author{
Jan Sunner \\ Department of Chemistry, Montana State University, Bozeman, Montana, USA
}

\begin{abstract}
Liquid secondary ionization mass spectra of solutions of alkali chlorides in glycerol were studied as a function of salt concentration. The experimental abundances of glycerol ions and of $\mathrm{Cs}^{+}(\mathrm{CsCl})_{n}$ cluster ions were successfully reproduced by assuming that most of the randomly distributed ions pair up with counterions shortly after impact. Further, it is considered that clustering (or proton transfer) reactions occur mainly between an ion that survives the pairing process and ion pairs (or basic analytes) in the immediate vicinity; however, some mixing undoubtedly occurs in the later stages of the desorption process. At the density of the original matrix, the range of proton transfer is calculated to be 5-15 $\AA$ and that of clustering approximately $25 \%$ shorter. These reaction distances are inversely correlated with the internal energy of the ejected ions. In general, liquid secondary ionization mass spectra of alkali chloride solutions can be seen to result from competitive ion-ion recombination reactions in the decaying matrix. Finally, from the abundances of cluster ions containing [glycerol $-\mathrm{H}^{-}$ions, it is estimated that approximately $1 \%$ of the glycerol molecules in the ejected volume are ionized in the collision cascade. (J Am Sac Mass Spectrom $1993,4,410-418$ )
\end{abstract}

$\mathrm{T}$ The formation of charged clusters is a ubiquitous phenomenon in all desorption ionization (DI) techniques. In solid and liquid secondary ionization mass spectrometry (SIMS), the mechanism of formation of such clusters has intrigued researchers for many years. Two competing viewpoints run through many years of literature: cluster formation by recombination in a gaseous phase, often called selvedge, or by ejection of clusters already present in the surface, known as "direct emission." It has proved difficult to make a clear choice between these alternatives. In contrast, it is well known that unimolecular dissociation of the clusters, after ejection from the surface, plays a large role in determining observed cluster distributions $[1,2]$.

The studies of alkali halides provide a good illustration of possible cluster formation processes. In solid SIMS, it was found that the $\left[\mathrm{M}(\mathrm{MX})_{n}\right]^{+}$clusters exhibited anomalous abundance distributions with enhanced abundances for some $n$ values [3-5]. These were interpreted in terms of the direct emission process [4,6]; however, the origin of the anomalies became clear when Standing and co-workers [1] found that no anomalies were observed in the mass spectra of

Address reprint requests to Jan Sunner. Department of Chemistry, Montana State University, Bozeman, MT 59717-0340.
CsI when a time-of-flight instrument was used as opposed to a magnetic sector instrument. The crucial difference between the two instruments is that the time-of-flight mass spectrometer shows the cluster distribution approximately $10^{-1} \mu \mathrm{s}$ after emission, whereas the sector instrument shows the distribution after approximately $10^{1} \mu$ s. It was thus clear that the anomalies did not originate with the cluster formation process but were due to subsequent unimolecular declustering [1]. Stability variations between different clusters are reflected in their unimolecular dissociation kinetics $[7,8]$. Collaborating support comes from the observation that the abundance anomalies are suppressed by collisional stabilization in a high-pressure source [9]. With respect to liquid SIMS, Miller and Theberge [10] and Wong and Röllgen [11] found that the abundance anomalies are strongly suppressed when the salt is dissolved in a liquid matrix (glycerol). It was concluded that the $\left[\mathrm{M}(\mathrm{MX})_{n}\right]^{+}$clusters have less internal energy when formed from a liquid matrix than from the solid [11]. However, this does not necessarily mean that the clusters leave the surface with less excitation energy; it is also possible that the $\left[\mathrm{M}(\mathrm{MX})_{n}\right]^{+}$ ions shed a glycerol (Gl) molecule very soon after ejection into the gas phase:

$$
\left[\mathrm{M}(\mathrm{MX})_{n}(\mathrm{Gl})\right]^{+} \rightarrow\left[\mathrm{M}(\mathrm{MX})_{n}\right]^{+}+\mathrm{Gl}
$$

Such a cooling effect of declustering was proposed [2] 
to explain the low internal energies of ions observed in molecular SIMS. The work cited above [1, 7-11] showed that unimolecular declustering is important in both solid and liquid SIMS; however, the mechanism of cluster formation remained uncertain.

A study of the many reports that support the alternative cluster formation process (i.e., recombination) shows that two very different physical pictures have been used. According to the first, usually applied to solid SIMS, the surface is essentially intact during desorption, and concepts like "surface-binding energy" and "passing through the surface" are meaningful [12]. This may be justified when desorption occurs on the $10^{-12}-\mathrm{s}$ time scale and is induced by momentum transfer in the collision cascade. Molecular dynamics simulations of solid SIMS have shown that recombination to form clusters still can occur when atoms that originally are nonadjacent happen to be ejected close together and with similar velocities $[13,14]$. The second picture, "bulk desorption," is very different and has been applied mainly to SIMS of liquids and frozen cryogenic gases. Here, it is considered that the matrix undergoes a bulk transformation to a gaseous state as a result of a "thermal spike." Recombination to form clusters is considered to occur in this high-pressure selvedge [15]. The concept of bulk desorption in liquid SIMS is consistent with several measurements of high neutral yields, approximately $10^{3}$ molecules per incident atom [16, 17]. From their extensive investigation into SIMS of frozen gases [18], Urbassek and Michl [19] arrived at a related "gas-flow" model. They considered that large, charged clusters are formed in a dense, supercritical flowing gas at the impact site as a result of "preferred solvation shells" around the ions [18]. These clusters subsequently cool by declustering in the vacuum. Gas-phase recombination has also been supported by others $[20,21]$.

A hot, very high-density gas with the small physical dimensions of the selvedge will have a very short lifetime. Thus, Michl and co-workers [18] argued that there is not enough time for three-body collisions to occur. Our own calculations also support that the analogy with clustering in a supersonic nozzle does not hold [22]. Indeed, Urbassek and Mich] [19] successfully modeled the gas flow at the impact site by using collision-free molecular flow.

The alternative to cluster formation by recombination at the surface or in the selvedge is "direct emission" $^{\prime \prime}$ of clusters. We may also consider two extreme pictures of this process. In the first, thermalization is extensive, and the energy of the matrix becomes nearly statistically distributed. Such cluster formation has been modeled using the Rice-Ramsperger-KasselMarcus (RRKM) theory [23]. In the other extreme, the cold matrix may be seen as "fracturing" into pieces (droplets). In this case, the excitation energy is very unevenly distributed among the different degrees of freedom. Examples include the "sudden perturbation" model in plasma desorption mass spectrometry [24] and the "bond-breaking" model $[4,25]$ in SIMS, in which alkali halide clusters are considered to result from direct emission of fragments of the original crystal. Molecular dynamics simulations do not seem to support such a notion, except in the sense that molecules may be ejected intact from metal surfaces [13]. Similar ideas of direct emission have been applied to liquid matrices. The driving force for a "fracturing" process could be a high-pressure gas pocket, formed as a result of the thermal spike underneath the surface $[26,27]$. The pressure is released in an explosive event. Vestal [27] proposed the lively analogy of a "splashing sauce." In a similar vein, Wong and Röllgen [26] proposed sputtering by "spraying." According to these ideas, bare analyte ions or clusters are formed as the ejected singly charged droplets (large clusters) shed all or most of their solvent molecules. These "droplet" models may be applied not only to liquid SIMS but also to other DI methods like laser desorption.

In our own work, we suggested a somewhat different explanation for the formation of charged solvent clusters. When the energy density in a matrix after an impact is high enough for the matrix to initiate a transition to a gaseous state but not high enough to actially form a gas, cluster formation must result [28]. Further, it must occur concurrently with the disintegration of the matrix. Thus, density variations gradually develop into isolated clusters. During this entropydriven, spinodal-like phase separation, the temperature drops sharply [28]. Several factors seem to influence the tendency of a matrix to undergo such a bulk desorption or spinodal transition, such as the energy of the bombarding particle, the shape and extent of the collision cascade, and the viscosity of the matrix [29].

In the present work, we analyze the change in the abundances of matrix and alkali chloride clusters in liquid secondary ionization mass spectra with changes in the concentration of the salt in a glycerol matrix. The results are interpreted in terms of cluster formation models.

\section{Experimental}

The experiments were performed with a VG 7070E-HF mass spectrometer with a VG 11-250J data system. The saddle-field fast-atom gun was operated at $8.0 \mathrm{kV}$ and 1.0 $\mathrm{mA}$ using xenon gas. The probe was fitted with a copper tip with a diameter of $6.5 \mathrm{~mm}$ and an angle between the line normal to the tip surface and the ion exit path of $20^{\circ}$. A magnet scan rate of $10 \mathrm{~s} /$ decade was used. The spectra used for this study are the average of the first three scans recorded after the sample was inserted into the ion source; however, the spectra were very stable over a time period of many minutes.

The chemicals were obtained commercially and used without further purification, except that glycerol was 
thoroughly degassed in an ultrasound bath prior to use. Samples were dissolved in the glycerol by stirring and in some cases by gentle heating to produce supersaturated solutions.

\section{Results and Discussion}

The best known liquid secondary ionization mass spectrum is probably that of neat glycerol. The base peak in that spectrum is protonated glycerol $\left(\mathrm{GlH}^{+}, m / z\right.$ 93). There are also a number of low-mass ions, notably at $m / z 75,57,45,31,29$, and 19. Most of these ions are probably formed as a result of gas-phase dissociations of $\mathrm{GlH}^{+}$. The "chemical noise" at nearly every integral mass is known to be due to radiation chemistry in the liquid matrix [30-32]. Finally, at high mass, there is a long sequence of cluster ions, $(\mathrm{Gl})_{n} \mathrm{H}^{+}$, observed up to $n>10$. We begin by taking a close look at the disappearance of the glycerol clusters and the simultaneous appearance of new clusters as the alkali chloride concentration is increased.

\section{Concentration Dependence of Liquid Secondary Ionization Mass Spectra of Glycerol Solutions of Alkali Chlorides}

The abundances of different cluster ions in the liquid secondary ionization mass spectra of alkali chlorides dissolved in glycerol were studied as a function of salt concentration. Previous studies of such spectra [9-11, 33] contain little concentration-dependent data. Figure 1 shows typical positive ion mass spectra, here of 1.0 and $10 \mathrm{~mol} \% \mathrm{CsCl}$ in glycerol. There are several groups of prominent ions. The ions of the neat glycerol spectrum are still present; however, the abundances, particularly of the glycerol clusters, are reduced. Further, there is a series of $\mathrm{Cs}^{+}(\mathrm{Gl}) \mathrm{m}$ ions (i.e., $\mathrm{Cs}^{+}$ions solvated by $m$ glycerol molecules). In another group of prominent peaks, the alkali ions are "solvated," also by one or several $\mathrm{CsCl}$ urits, $\mathrm{Cs}_{s}{ }^{+}\left(\mathrm{Cs} \mathrm{Cl}_{n}(\mathrm{Gl})_{m}\right.$. At higher salt concentrations, this sequence of $\mathrm{Cs}^{+}(\mathrm{CsCl})_{n}$ ions extends to high $n$. Finally, there are a number of ions in the spectrum with composition $\mathrm{Cs}^{+} \mathrm{CCs}^{+}[\mathrm{G}]-$ $\left.\mathrm{H}^{-}\right)(\mathrm{CsCl})_{n}(\mathrm{Gl})_{m}$ (i.e., where one chloride ion is substituted by a glyceroxide anion-the deprotonated glycerol molecule).

The dependence of ion abundances on salt concentration was studied for a number of alkali chlorides as well as for mixtures of chlorides. Different alkali ions give very similar results; however, the solubility is the largest for the cesium salt. Therefore, these spectra can be studied over the widest concentration range and only the cesium chloride results are shown here. Figure 2 shows the behavior of the main glycerol ions. In these semilogarithmic graphs, it is striking that the slopes of the fitted lines are much larger for the glycerol clusters than for $\mathrm{GIH}^{+}$and, in particular, for the glycerol fragment ions. We previously observed this

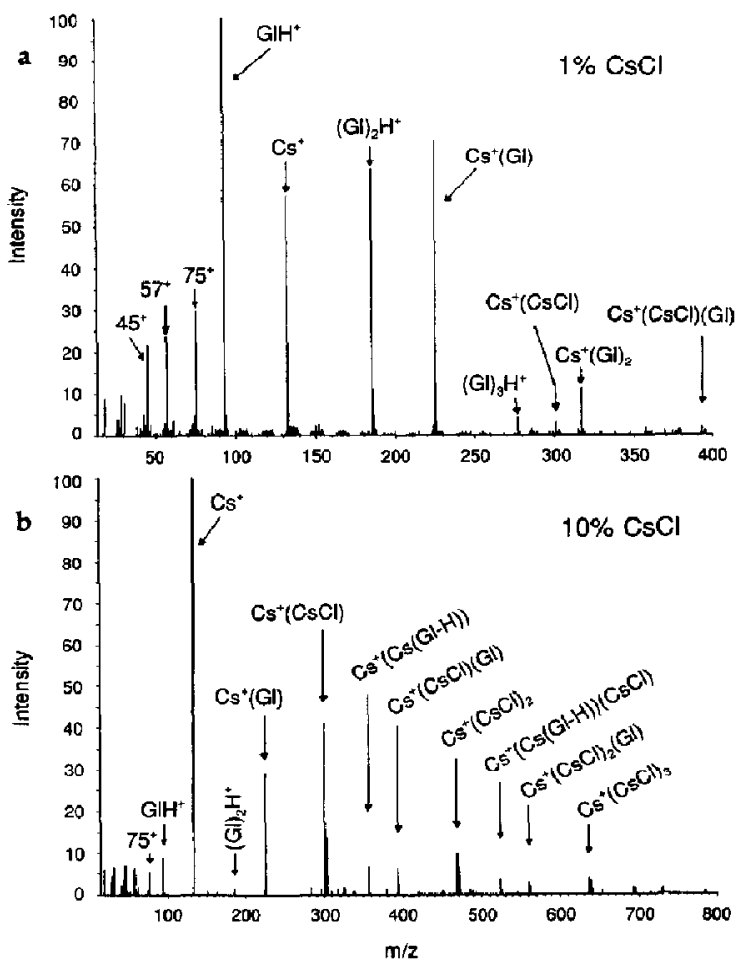

Figure 1. Liquid secondary ionization mass spectrum of (a) 1.0 mol\% and (b) $10 \mathrm{~mol} \%$ solution of $\mathrm{CsCl}$ in glycerol.
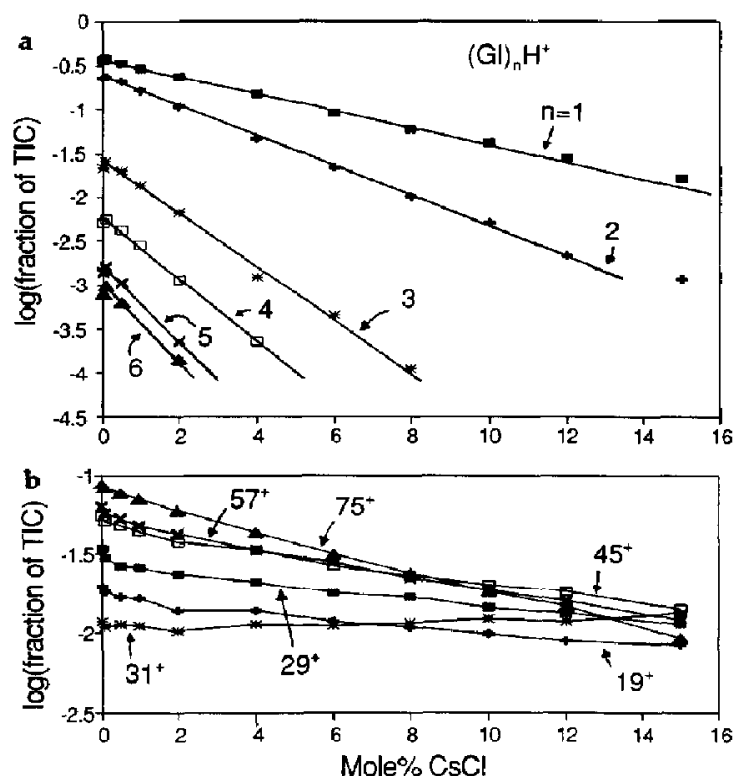

Figure 2. Abundances of principal glycerol ions as a function of the concentration of $\mathrm{CsCl}$ in the matrix. (a) $(\mathrm{Cl})_{n} \mathrm{H}^{+}$ions with $n=1-6$; (b) major glycerol fragment ions. Note the difference in scale; TIC, total ion current. 
phenomenon when adding diethanolamine to glycerol [34]. A possible explanation would seem to be that for some unknown reason, the average internal excitation energy of ejected glycerol ions and clusters increases as salt is added, leading to more extensive fragmentation; however, a better explanation is that "ionization transfer" from glycerol to alkali ions, $\mathrm{M}^{+}$.

$$
\mathrm{GlH}^{+} \text {ions } \stackrel{1 \rightarrow Q}{\longrightarrow} \mathrm{M}^{+} \text {ions }
$$

occurs during the desorption event, where $1 \rightarrow \mathrm{g}$ indicates that this reaction occurs during the transition from a liquid to a gas. If the extent of reaction 2 is the largest for the large glycerol cluster ions and the smallest for the glycerol fragment ions, then this would qualitatively explain the trend shown by the curves in Figure 2. Reaction 2 is analogous to the proton transfer observed, when energetically favorable, for basic analytes B dissolved in glycerol [35],

$$
\mathrm{GlH}^{+} \text {ions }+\mathrm{B} \stackrel{\mathrm{l \rightarrow g}}{\longrightarrow} \mathrm{BH}^{+} \text {ions }
$$

For salts, gas-phase proton transfer to an alkali halide ion pair is exothermic [33]:

$$
\mathrm{GlH}^{+}+\mathrm{MX} \stackrel{\mathrm{g}}{\longrightarrow} \mathrm{M}^{+}(\mathrm{Gl})+\mathrm{HX}
$$

However, the same products would result from the association reaction

$$
\text { (Gl) }{ }_{m} \mathrm{H}^{+}+\mathrm{MX} \stackrel{\mathrm{g}}{\longrightarrow}(\mathrm{Gl})_{m} \mathrm{H}^{+}(\mathrm{MX})
$$

followed by intracluster proton transfer and evaporation of the volatile HX molecule,

$$
(\mathrm{Gl})_{m} \mathrm{H}^{+}(\mathrm{MX}) \stackrel{\mathrm{g}}{\longrightarrow} \mathrm{M}^{+}(\mathrm{Gl})_{m}+\mathrm{HX}
$$

During a liquid-to-gas transition, the ions are solvated by a disintegrating glycerol solvation shell. Thus, it is difficult to make a distinction between reactions 4 and 5. It should be noted that substituting $\mathrm{X}^{-}$with a $[\mathrm{Gl}-\mathrm{H}]^{-}$ion in reactions 4 and 5 results in the same ionization transfer from $\mathrm{GlH}^{+}$to $\mathrm{M}^{+}$.

\section{Analysis of Liquid Secondary Ionization Mass Spectra of Alkali Chlorides in Glycerol Using the Droplet Model}

According to the droplet model, reaction 2 occurs within a charged droplet ejected from the surface. This is represented by reaction $5 b$ with a large $m$. In subsequent dissociations, only neutrals are shed from the cluster to leave behind a product ion. If by chance a charged droplet does not contain an alkali ion, a glycerol ion will result; however, if the droplet does contain an alkali ion, reaction $5 \mathrm{~b}$ occurs, and an " $\mathrm{M}^{+}$ion" is detected instead. The trends of the slopes in Figure 2 can then be explained if the following correlation holds: Larger droplets tend to end up as large glycerol clus- ters, whereas smaller droplets tend to end up as $\mathrm{GlH}^{+}$ or glycerol fragment ions. Because a larger droplet has a higher probability of containing at least one alkali ion, the formation of large alkali-free droplets - the presumed precursors of $\mathrm{Gl}_{n} \mathrm{H}^{+}$clusters-becomes very unlikely as the salt concentration increases.

Within the droplet model, it is not at all obvious why there should be a correlation between droplet size and the internal energy of the resulting ion or cluster. Still, this idea is developed here quantitatively, and we show that the observed spectra are reproduced very well. However, it is not suggested that this supports the droplet model for liquid SIMS; rather, we argue that the "droplets" must be reinterpreted in terms of proton transfer and clustering "volumes" in the disintegrating matrix.

The following assumptions are made: (1) the average composition of all the droplets, when formed, is the same as the bulk composition of the matrix; (2) the composition of individual droplets is statistically distributed; (3) the droplets, after formation, shed only solvent molecules and other neutrals; and (4) only singly charged droplets contribute to the mass spectra. Thus, whereas the probability that a droplet contains $n$ ions of the same charge must be calculated, the probability that the same droplet contains $n-1$ counterions is always 1 .

Assumption 1 above needs to be carefully considered. It would seem that evaporation of matrix molecules, coupled with slow diffusion of analyte in the viscous matrix, must result in a considerable enrichment of salt near the surface; however, there is evidence to the contrary. First, at the high primary beam intensities used in fast-atom bombardment, the rate of sputtering roughly equals the rate of evaporation [36]. In such a case, the surface concentration would be approximately double the bulk concentration. Second, Kriger et al. [37], using imaging SIMS, showed that non-surface-active analytes concentrate along the periphery of the droplet. These investigators explained that as a concentrated salt solution is formed in the immediate vicinity of the surface, the surface tension increases. As a result, excess salt in the surface is efficiently transported from the center to the periphery of the droplet. Third, the sum abundance of the salt ions in the spectra is linear with salt concentration up to a point close to the solubility limit. A conservative estimate therefore is that the salt concentration probed in the liquid SIMS experiment is less than twice that of the bulk concentration. Therefore, the assumption that the surface and the bulk concentrations are equal seems not to be grossly in error.

The sizes of the charged droplets that are the assumed precursors to the different glycerol ions are now estimated from the results in Figure 2 . The procedure is illustrated using the $(\mathrm{Gl})_{4} \mathrm{H}^{+}$clusters. Assume that the corresponding precursor droplets originally contain $d$ molecules. The probability $p_{\mathrm{g}}$ that a droplet will not contain a cesium ion [and thus result in the 
$(\mathrm{Gl})_{4} \mathrm{H}^{+}$cluster] is

$$
p_{\mathrm{g}}=\left(1-x_{\mathrm{Cs}}\right)^{d}
$$

where $x_{C_{6}}$ is the mole fraction of $\mathrm{CsCl}$. Equation 6 predicts a decrease in the $\left(\mathrm{Gl}_{4} \mathrm{H}^{+}\right.$abundance with increasing $x_{\mathrm{Cs}}$. It is seen in Figure 2 that the abundance of the $\left(\mathrm{Gl}_{4} \mathrm{H}^{+}\right.$clusters decreases by a factor of 2.2 for every $1 \mathrm{~mol} \%$ increase in $x_{\mathrm{CsCl}}$. Setting the parent droplet size $d$ at 78 in eq 6 gives an almost perfect fit to this line. Similarly, $d$ was calculated for the other glycerol cluster and fragment ions. The fitted lines are not shown; they nearly overlap the lines connecting the data in Figure 2. The line for fragment $31^{+}$has a positive slope and is excluded here. The calculated $d$ values range from 6 for the high-energy fragment $29^{+}$to 16 for $75^{+}, 22$ for $93^{+}$, and approximately 100 for the largest glycerol clusters. Of course, these are average values $d_{\text {aver }}$ each glycerol ion should be associated with a range of $d$ values. By assuming a Gaussian distribution with a standard deviation of $d_{\text {ave }} / 3$ and normalizing relative areas to reflect the abundances of the respective ions in neat glycerol spectra, the approximate droplet-size distribution curves in Figure 3 are obtained. The three lower curves show the results for glycerol fragments $\left(\mathrm{F}_{\mathrm{G}}^{+}\right), \mathrm{GH}^{+}$, and $\mathrm{G}_{2} \mathrm{H}^{+}$, respectively. The sum droplet, or reaction volume, size distribution shows a shoulder above $n=20$. Assuming that the real distribution should be smooth, the standard deviation should not be chosen smaller than $d_{\text {ave }} / 3$ because the shoulder is then intensified. If, on the other hand, a larger standard deviation is chosen, then the "sum" curve evens out and broadens somewhat, but it retains its overall shape. Thus, the "sum" curve in Figure 3 should approximate the overall droplet or reaction volume (see below), size distribution in glycerol under our experimental conditions. The dashed line shows that the cluster abundances

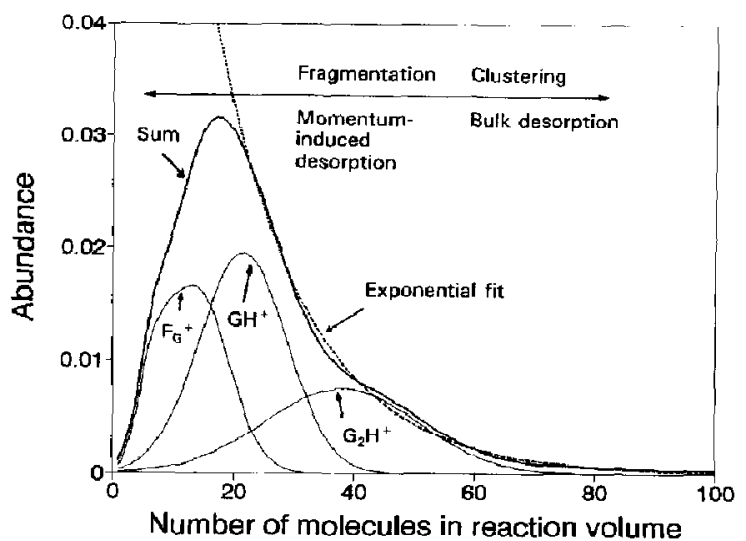

Figure 3. Abundances of "droplets" as a function of the number of molecules in the droplet (see text). The droplets can also be interpreted as "reaction volumes" [i.e., local volumes within which the ionization transfer $\left(\mathrm{GlH}^{+}\right.$ta $\left.\mathrm{Cs}^{+}\right)$(reaction 2) occurs]. closely follow an exponential decay above $n=25$. It is seen that the abundance is halved as $n$ increases by approximately 10 . Assuming spherical droplets of uniform density equal to that of pure glycerol, this corresponds to a rather sharp falloff between 9 and $11 \AA$.

We now turn our attention to the $\mathrm{Cs}^{+}(\mathrm{CsCl})_{n}(\mathrm{Gl})_{m}$ ions. These are abundant in the spectra, particularly at higher salt concentrations. Figure 4a shows the experimental abundances of such clusters with different $n$ summed over $m$. For example, the points for $n=2$ show the sum abundance of the ions that contain exactly three $\mathrm{Cs}^{+}$ions. The probabilities that an original droplet of size $d$ will contain $n+1$ cesium ions are given by

$$
p_{k}=x_{\mathrm{Cs}}^{k} *\left(1-x_{\mathrm{Cs}}\right)^{d-k} *\left(\begin{array}{l}
d \\
k
\end{array}\right)
$$

where $\left(\begin{array}{l}d \\ k\end{array}\right)$ is a binomial coefficient, and $k=n+1$ Equation 7, convoluted over the distribution in Figure 3 , reproduces the sum abundance of all $\mathrm{Cs}^{+}(\mathrm{CsCl})_{n}(\mathrm{Gl})_{m}$ clusters as a function of $x_{\mathrm{Cs}}$ very well, as expected; however, the abundances of the higher $n$ clusters become severely overestimated. For example, at 15 mol\% CsCl, the abundance for $n=4$ is
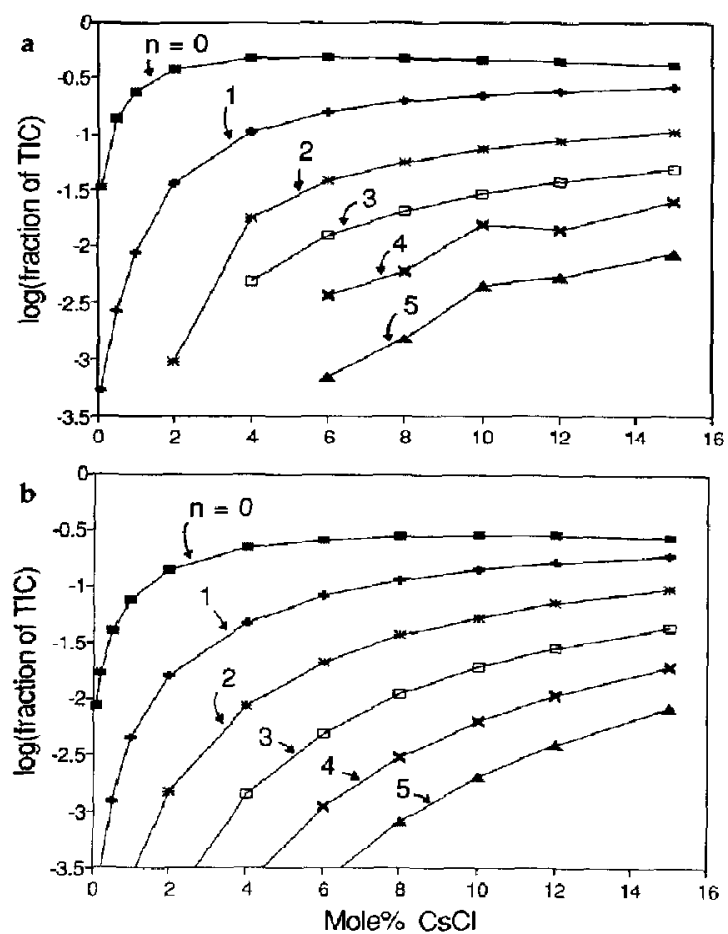

Figure 4. (a) Experimental abundances of $\mathrm{Cs}^{+}(\mathrm{CsCI})_{n}(\mathrm{GI})_{m}$ for $n=0-5$ and summed over $m$. (b) Abundances of the same clusters as in a calculated with 7 . The "droplet" distribution used was that shown in Figure 3, except that the "droplet" sizes were reduced to $40 \%$ : TIC, total ion current. 
predicted to be slightly higher than that for $n=1$. In contrast, the experimental $n=1$ abundance was eight times larger. The droplet sizes therefore had to be reduced to $40 \%$ of those shown in Figure 3 to obtain a good fit. The relative abundances of the cluster ions with different $n$ were then well reproduced (see Figure $4 \mathrm{~b}$ ); however, the total abundance of such ions is now underestimated. This difference in droplet size is explained below in lonic Clustering and Proton Transfer Distances in the Decaying Matrix.

Within each group of $\mathrm{Cs}^{+}(\mathrm{CsCl})_{n}$ ions, there are clusters solvated by a different number $m$ of glycerol molecules. Figure 5 illustrates this for the $\mathrm{Cs}^{+}(\mathrm{CsCl})_{2}$ $(\mathrm{Gl})_{m}$ clusters. It is seen that the extent of clustering with glycerol within the group decreases rapidly with increasing salt concentration. Figure $5 b$ shows the same data as in Figure 5a but with the total abundances (i.e., summed over $m$ ) normalized to $100 \%$ at each concentration. It is seen that the behavior is very similar to that of the glycerol ions in Figure 2 (i.e., the abundances at different $m$ decay exponentially with increasing $\mathrm{CsCl}$ concentration). The same explanation is applicable: The cooler $\mathrm{Cs}^{+}(\mathrm{CsCl})_{2}$ clusters (i.e., those detected with several glycerol molecules) are derived from larger droplets. Thus, they are also more likely to add on an additional $\mathrm{CsCl}$ ion pair. Calculations based on this assumption satisfactorily reproduced the ion abundances in Figure 5; however, the calculation in-
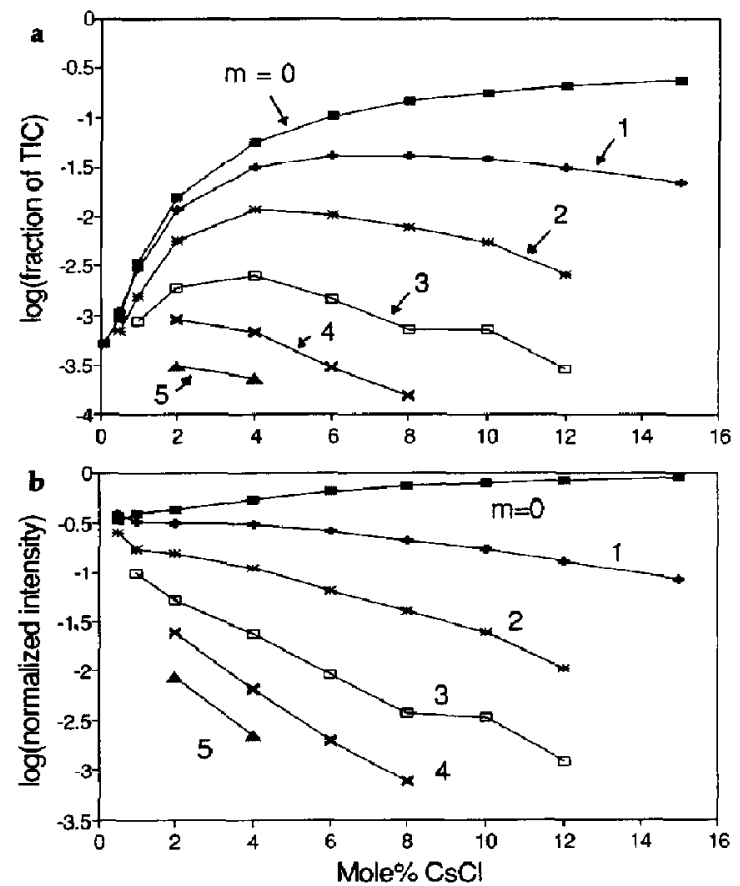

Figure 5. Experimental abundances of $\mathrm{Cs}^{+}(\mathrm{CsCl})_{2}(\mathrm{Gl})_{m}$ cluster ions for in $=0-5$ as a function of $\mathrm{CsCl}$ concentration in glycerol; (a) absolute abundances; (b) normalized abundances; TIC, total ion current. volved fitting a number of $d$ values to observed $m$ values and the results are not shown.

We now turn to the presence of $[\mathrm{Gl}-\mathrm{H}]^{-}$ions in the liquid secondary ionization mass spectra. Judging from the negative ion spectrum of glycerol, $[\mathrm{Gl}-\mathrm{H}]^{-}$ is the main negative ion formed as a result of ionization reactions in the collision cascade. Figure 6 shows the observed abundances of $\mathrm{Cs}^{+}(\mathrm{CsCl})_{n}\left(\mathrm{Cs}^{+}[\mathrm{Gl}-\right.$ $\left.\mathrm{H}^{-}\right)(\mathrm{Gl})_{m}$ cluster ions for $n=0-2$ summed over $m$. A comparison with Figures 2 and 4 shows that the concentration dependence is very different. We were interested to see whether this behavior could also be accounted for by the droplet model. The simplest assumption is that $[\mathrm{Gl}-\mathrm{H}]^{-}$(together with an arbitrary counterion $\mathrm{P}^{+}$) can be treated as any other analyte with a mole fraction of $x_{\mathrm{h}}$. The probability $p_{n}^{\prime}$ of a droplet containing the ions in a $\mathrm{Cs}^{+}\left(\mathrm{Cs}^{+}[\mathrm{Gl}-\mathrm{H}]^{-}\right)$ $(\mathrm{CsCl})_{n} \mathrm{Gl}_{m}$ cluster is obtained as the product of the probabilities that (1) the droplet contains a [Gl $\mathrm{H}^{-} \mathrm{P}^{+}$ion pair; (2) the counterion $\mathrm{P}^{+}$is a $\mathrm{Cs}^{+}$and not a $\mathrm{GlH}^{+}$ion, which would recombine with the $[\mathrm{Gl}-$ $\mathrm{H}^{-}$ion to leave neutrals; (3) the droplet contains $n+1$ additional $\mathrm{Cs}^{+}$ions. Setting the mole fraction of the $\mathrm{GlH}^{+}$ions to $x_{\mathrm{h}}$ we arrive at

$$
\begin{aligned}
p_{n}^{\prime}= & \left(1-x_{\mathrm{h}}\right)^{\mathrm{d}-1} * x_{\mathrm{h}} * d * r_{\mathrm{Cs}} \\
& * x_{\mathrm{Cs}}^{n+1} *\left(1-x_{\mathrm{Cs}}\right)^{d-n-2} *\left(\begin{array}{l}
d-1 \\
n+1
\end{array}\right)
\end{aligned}
$$

where

$$
r_{\mathrm{Cs}}=\left[x_{\mathrm{Cs}} /\left(x_{\mathrm{Cs}}+x_{\mathrm{h}}\right)\right]
$$

and $\left(\begin{array}{l}d-1 \\ n+1\end{array}\right)$ is a binomial coefficient. The dashed curves in Figure 6 show the ion currents calculated with eq 8. For this calculation, we used $x_{\mathrm{h}}=0.01$ and the smaller droplet distribution that was used for the calculation of $\mathrm{Cs}^{+}(\mathrm{CsCl})_{n}(\mathrm{Gl})_{m}$ cluster abundances of Figure 4b. Any significant changes in either the droplet distribution or in $x_{\mathrm{h}}$ destroy the agreement seen in Figure 6 . The $1.0 \mathrm{~mol} \%$ concentration of $[\mathrm{Gl}-\mathrm{H}]^{-} \mathrm{P}^{+}$

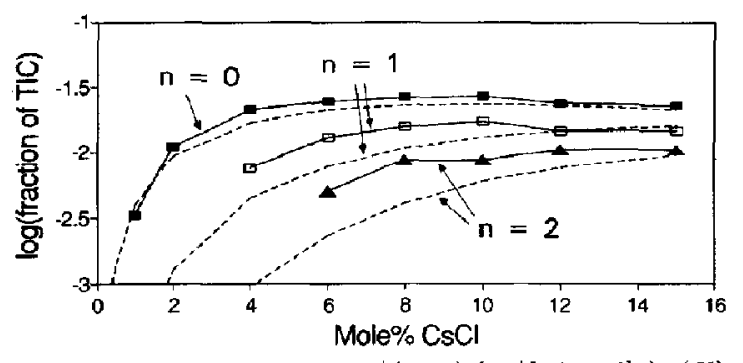

Figure 6. Abundances of $\mathrm{Cs}^{+}(\mathrm{CsCl})_{n}\left(\mathrm{Cs}^{+}[\mathrm{Gl}-\mathrm{H}]^{-}\right)(\mathrm{Gl})_{m}$ cluster ions for $n=0-2$ summed over $m$. Solid lintes, experimental abundances; dashed lines, abundances calculated from 8 using a mole fraction of 0.01 for the $\left[\mathrm{Gl}-\mathrm{H}^{-}\right.$concentration; TIC, total ion current. 
must be considered a rough estimate only. Still, it is a figure of considerable interest as an experimentally based estimate of the extent of ionization in the collision cascade in liquid SIMS. It should be noted, however, that the ion yield is much lower because of extensive recombination.

Further refinements of the calculations for Figure 6 within the "droplet" model were found to be of doubtful value. The best value for $x_{h}$ remained approximately $1 \%$.

\section{Ionic Clustering and Proton Transfer Distances in the Decaying Matrix}

We have seen that the liquid secondary ionization mass spectra of alkali chlorides, at varying concentrations, in glycerol can be explained within the droplet model: After a droplet is separated from the rest of the matrix and ejected into the vacuum, it sheds all, or nearly all, neutral matrix molecules. Whether the droplet is detected as a glycerol ion or a Cs${ }^{+}(\mathrm{CsCl})_{n} \mathrm{Gl}_{m}$ or $\mathrm{Cs}^{+}\left(\mathrm{Cs}^{+}[\mathrm{Gl}-\mathrm{H}]^{-}(\mathrm{CsCl})_{n} \mathrm{Gl}_{m}\right.$ cluster ion depends on what ions were originally present in the droplet. Our calculations strongly support that this is a matter of chance. This is to be expected if the ions are randomly distributed in the matrix at the time of impart. There was, however, an inconsistency in that modeling the increased abundance of the higher cesium ion clusters required smaller droplets than did modeling the decreasing abundance of the larger glycerol ions. This cannot be explained by an error in $x_{C s}$ and is hard to reconcile with a droplet model; however, there is a more serious problem.

The evaporation of one glycerol molecule requires approximately $17 \mathrm{kcal} / \mathrm{mol}$. Most of that energy is taken from internal degrees of freedom in the daughter cluster. If the parent cluster is initially cold, there is not enough energy for a second molecule to evaporate. For several successive evaporations to occur, the parent clusters must have a high initial excitation energy. We recently made RRKM calculations of the unimolecular dissociations of $(\mathrm{Gl})_{n} \mathrm{H}^{+}$clusters with $n$ up to 10 [38]. Even considering the uncertainties of such calculations, the result is unequivocal. To achieve substantial desolvation, the internal excitation energy of the clusters must be very high; however, the first dissociation reactions are then very fast. The separation of a large cluster or droplet from the matrix involves breaking a multitude of intermolecular hydrogen bonds and other interactions. It may be estimated that this process takes a minimum of $10^{-11} \mathrm{~s}$; however, the RRKM calculations show that most of the molecules would leave the cluster well before $10^{-11} \mathrm{~s}$. I'hus, these dissociation reactions cannot be conceptually separated from the disintegration of the matrix. After $10^{-11} \mathrm{~s}$, but before mass analysis in the spectrometer, RRKM predicts that a maximum of only one to two glycerol molecules are shed from any $\left(\mathrm{Gl}_{10} \mathrm{H}^{+}\right.$cluster. Thus, the cluster distribution observed in the liquid sec- ondary ionization mass spectrum of glycerol should be rather close to the distribution of clusters ejected from the surface. "What you see is (almost) what you had." This result is also intuitively very reasonable.

Because the droplet model seems to be fundamentally flawed, the question is how to interpret the relative success of the calculations above. First, we deal with the possibility that the clusters are already present as aggregates in the matrix prior to impact. We believe that this possibility is excluded by the following two observations. First, mixed alkali ion clusters are found in liquid secondary ionization mass spectra with a near-statistical distribution [39]. Because there is plenty of time between projectile impacts to establish a "preformed" ion cluster distribution, the abundances of aggregates in the matrix should be determined largely by their relative stabilities. It is almost inconceivable that some clusters would not be considerably more stable than others; however, if the clusters are formed very quickly, their composition will be determined largely by what ions happen to be close together in the matrix prior to impact. The second observation is that the extent of clustering of $\mathrm{M}^{+}$ $\left(\mathrm{M}^{+} \mathrm{X}^{-}\right)$, with glycerol decreases exponentially with increasing salt concentration (Figure 5b), as illustrated by the change in the ratio of, for example, $m=2$ to $m=0$ in Figure $5 b$. It is difficult to understand why the presence of another salt or cluster ion in the vicinity of a preformed $\mathbf{M}^{+}\left(\mathbf{M}^{+} \mathbf{X}^{-}\right)_{2}$ cluster would substantially increase the internal excitation energy of the ejected $\mathrm{M}^{+}\left(\mathrm{M}^{+} \mathrm{X}^{-}\right)_{2}$ cluster. Thus, no dependence of $m$ on $\mathrm{CsCl}$ concentration is expected, at least at low concentrations. For the same reason, the exponential decay for the glycerol ions (Figure 2) becomes very difficult to explain. Thus, the evidence is that ejection of "preformed" clusters is not important. Further support comes from the observation that atom bombardment inhibits crystallization [40]

From the discussion above, it is concluded that because the alkali chloride clusters are formed neither before impact nor after ejection, they must be formed during the disintegration of the matrix. During this process, the density of the matrix decreases, and the temperature (initially) increases. Therefore, the dielectric constant will decrease [41], and the formation of ion pairs and ionic clusters becomes thermodynamically strongly favored. Because of the long-range forces involved, the tendency toward a gradual pairing of charges is very strong. The result is the formation of contact ion pairs. The fact that the ionic yield is only approximately $0.1[26,42]$ shows that only a smail fraction of the initially solvated ions survives recombination. l'o simplify the discussion, it is considered that this ion pairing occurs first. The "droplets" used in the modeling can then be thought of as spherical volumes surrounding each of the few surviving ions. As in the droplet model, the fate of the central ion is determined by what ion pairs happen to be present within the volume; however, the reactions are now considered to 
occur in the disintegrating matrix. In the case of ionization transfer from glycerol to alkali ions (reaction 2), these "proton transfer volumes" range from approximately 6 to 100 glycerol molecules (see Figure 3). At the original density of the matrix, this corresponds to radii of 5-15 $\AA$. Thus, a protonated glycerol molecule must be this far away from the nearest alkali/chloride ion pair to have a 50\% chance to survive (i.e., not to proton transfer to the chloride ion). Once proton transfer occurs, the cesium ions take over as the local, "surviving" charge, and other nearby $\mathrm{Cs}^{+} X^{-}$ion pairs will cluster with that ion; however, the radii of these "ionic clustering volumes" are approximately $25 \%$ smaller than the "proton transfer volumes," as evidenced by the smaller droplets needed to explain the cluster distributions in Figure 4. This is reasonable because of the high mobility of the proton. Thus, the difficulty with the different droplet distributions is seen to disappear. It should be noted that the reactive distances quoted above would decrease by a factor of only 1.26 if the salt concentration sampled at the surface is not equal to but double that of the bulk.

The "reactions volumes" considered above are not necessarily contiguous. It is certainly likely that during the latter part of the desorption process, some mixing occurs (i.e., there are occasional collisions between ions and molecules that were more distant in the matrix before impact). It is significant, however, that the "reaction" distances $r_{n}$ are not very large; at $9 \AA$, two ions are separated by only one glycerol molecule. Because neighboring ions and molecules are very likely to react, it seems that mixing cannot be very important. The simplest picture within which the present data can be explained seems to be one of a near-homogeneous, near-thermal bulk liquid-to-gas transition $[15,18,21$, 28], where neighbors would tend to remain neighbors during the desorption process. Indeed, in molecular dynamics simulations of the bulk desorption process $[43,44]$, most collisions are seen to occur between molecules that were originally neighbors in the matrix. The distance calculations in the present studies then show that most reactions occur between molecules that were neighbors in the original matrix. For proton transfer to basic analytes, and for the formation of the largest ionic clusters, more distant neighbors also become important.

According to the model presented here, the liquid secondary ionization mass spectra of alkali halides may be pictured as resulting from competitive ion-ion recombination reactions in the decaying matrix. For example, the "ionization transfer" from glycerol to alkali ions (reaction 2) can be written as

$$
\mathrm{GlH}_{\mathrm{g} \downarrow}^{+}+\mathrm{X}_{\mathrm{g} \downarrow}^{-}+\mathrm{M}_{\mathrm{g} \downarrow}^{+} \stackrel{\mathrm{l} \rightarrow \mathrm{g}}{\longrightarrow} \mathrm{Gl}+\mathrm{HX}+\mathrm{M}_{\mathrm{g} \downarrow}^{+}
$$

where the subscripts $g \downarrow$ remind us that the ions in particular experience a gradual decrease in the degree of solvation and an associated increase in the strength of the coulombic forces. Reaction 9 constitutes a competition between (1) ion pair formation involving $X^{-}$ and $\mathrm{GlH}^{+}$(reaction 9a), leading to proton transfer and ejection of an $\mathbf{M}^{+}$ion; and (2) ion pair formation involving $X^{-*}$ and $\mathrm{M}^{+}$(reaction 9b), leading to the ejection of a glycerol ion. The outcome depends on the statistical distributions in the relative positions of the solvated ions prior to impact.

The dominant ionization reaction in liquid SIMS is the protonation of basic analytes $\mathrm{B}$ to give intense $\mathrm{BH}^{+}$ peaks in the spectra. In a previous study, we presented a kinetic scheme that successfully reproduced the matrix and analyte ion abundances for solutions of diethanolamine in glycerol [34]. The model and physical picture presented in this study can also be applied to such systems with the same degree of success. Thus, analyte protonation in liquid SIMS can be seen as the result of proton transfer from $\mathrm{GlH}^{+}$, formed in the collision cascade, to any basic analyte that happens to be within approximately $10 \AA$ of the glycerol ion. The evidence is that this reaction occurs during the decomposition of the matrix.

\section{Conclusions}

The abundances of alkali chloride clusters, $\mathrm{M}^{+}(\mathrm{MCl})_{n}$ (Gl) ${ }_{n,}$ in liquid secondary ionization mass spectra of alkali chloride solutions in glycerol are given by the probability that $n \mathrm{M}^{+}$ions are found within a radius $r_{n}$ of approximately $7 \AA$ of a central alkali ion, assuming a random distribution of solvated ions prior to projectile impact. Similarly, the abundances of glycerol ions depend on the probability that no $\mathrm{M}^{+}$ions are present within a radius of approximately $9 \AA$ of $\mathrm{GlH}^{+}$ ion that is formed in the collision cascade. These results can be explained by desorption being due to a near-homogeneous liquid-to-gas transition, where neighbors tend to remain neighbors during the desorption process. The dominant ionic reaction is ion-ion recombination, which leaves only an occasional survivng charge among the ejected molecules. As analyte is added to the matrix, ionization transfer from matrix to analyte as well as formation of involatile alkali halide clusters result from further ion-ion recombination involving ions in the immediate vicinity of a surviving charge. Protonation of basic analytes in liquid SIMS is explained within the same picture. Finally, it is estimated that $1 \%$ of the glycerol molecules are ionized in the collision cascade.

The main importance of the present results is in the conclusion that neighbor interactions are all important to the chemistry during the desorption process. For example, there is presently much interest in laser desorption and in using that technique to detect noncovalent complexes [45]. Because of the fundamental thermodynamic nature of spinodal-like transitions from condensed phase to gas [28], and the evidence of 
extensive thermalization, it seems very likely that the desorption processes resulting from laser irradiation and from bombarding particles are closely related. Thus, it also seems likely that neighbor-neighbor interactions dominate the ionic chemistry during laser desorption as well. This is very encouraging for the outlook of detection of noncovalent complexes using laser desorption. As in liquid SIMS, the main problem would seem to be the prevention of dissociation of the complex prior to desorption. Further, in the present study, we made the reasonable assumption that alkali and halide ions do not dissociate from a cluster; however, in the more general case of noncovalent complexes, a good understanding of the competitive dissociation dynamics of complex and matrix is also needed.

\section{Acknowledgments}

We thank Jentaie Shiea and Joe Sears for assistance with the experiments. This work was supported by a grant from MONTS.

\section{References}

1. Ens, W.; Beavis, R.; Standing, K. G. Phys. Rev. Lett. 1982, 50, 27

2. Busch, K. L.; Hsu, B. H.; Xie, Y. X.; Cooks, R. G. Anal. Chem. $1983,55,1157$.

3. Taylor, J. A.; Rabalais, J. W. Surf. Sci. 1978, 74, 229.

4. Campana, J. E.; Barlak, T. M.; Colton, R. J.; DeCorpo, J. J.; Wyatt, J. R; Dunlap, B. I. Phys. Reo. Lett 1981, 47, 1046.

5. Barlak, T. M.; Campana, J. E.; Wyatt, J. R.; Colton, R. J. I. Phys. Chem. 1983, 87, 3441.

6. Dunlap, B. I. Surf. Sci. 1982, 121, 260.

7. Campana, J. E.; Green, B. N. J. Am. Chem. Soc. 1984, 106, 531.

8. Hwang, H. J.; Sensharma, D. K.; El-Sayed, M. A. J. Phys. Chem. 1989, 93, 5012.

9. Callahan, I. H.; Colton, R. J.; Ross, M. M. Int. J. Mass Spectrom. Ion Processes 1989, 90, 9.

10. Miller J. M.; Theberge, R. Org. Mass Spectrom. 1985, 20, 600.

11. Wong, S. S.; Röllgen, F. W. Int. I. Mass Spectrom. Ion Processes 1986, 70, 135.

12. Honda, F.; Lancaster, G. M.; Fukuda, Y.; Rabalais, J. W. J. Chem. Phys. 1978, 69, 4931.

13. Winograd, N.; Garrison, B. J. In Ion Spectroscopies for Surface Analysis; Czanderna, W.; Hercules, D. M., Eds.; Plenum: New York, 1991.

14. Garrison, B. J. Int. I. Mass Spectrom. Ion Processes 1983, 53, 243.

15. Lancaster, G. M.; Honda, F.; Fukuda, Y.; Rabalais, J. W. I. Am. Chem. Soc. 1979, 101, 1951.
16. Wong, S. S.; Röllgen, F. W.; Manz, I.; Przybylski, M. Biomed. Mass Spectrom. 1985, 12, 43.

17. Jiang, I. F.; Barofsky, E.; Barofsky, D. F. Proceedings of the 36th ASMS Conference on Mass Spectometry and Allied Topics; San Francisco, CA, 1988; p 1209.

18. David, D. E; Magnera, T. F.; Tian, R.; Stulik, D.; Michl, J. Nucl. Instrum. Methods 1986, B14, 378.

19. Urbassek, H. M.; Michl, J. Nucl. Instrum. Methods 1987, B22, 480.

20. Cox X. B. III; Linton, R. W.; Bursey, M. M. Int. J. Mass Spectrom, Ion. Processes 1983, 55, 281.

21. Pachuta, S. J.; Cooks, R. G. Chem. Rev. 1987, 87, 647.

22. Sunner, J., unpublished results.

23. King, B. V.; Tsong, I. S. T.; Lin, S. H. Int. J. Mass Spectrom Ion Processes 1987, 78, 341 .

24. Krueger, F. R. Z. Naturforsch. 1983, 38a, 385.

25. Campana, J. E.; Dunlap, B. I. Int. I. Mass Spectrom. Ion Processes 1984, 57, 103.

26. Wong, S. S.; Röllgen, F. W. Nuct. Instrum. Methods 1986, B14, 436.

27. Vestal, M. L. Mass Spectrom. Rev. 1983, 2, 447.

28. Sunner, J.; Ikonomou, M. G.; Kebarle, P. Int. I. Mass Spectrom. Ion Processes 1988, 82, 221.

29. Shiea, J. T.; Sunner, J. Int. J. Mass Spectrom. Ion Processes 1990, $96,243$.

30. Field, F. H. J. Phys. Chem. 1982, 86, 5115.

31. Keough, T.; Ezra, F. S.; Russell, A. F.; Pryne, J. D. Org. Mass Spectrom. 1987, 22, 241.

32. Caldwell, K. A.; Gross, M. L. Proceedings of the 38th ASMS Conference on Mass Spectrometry and Allied Topics; Tucson, AZ, 1990; p 180.

33. Keough, T. Anal. Chem. 1985, 57, 2027.

34. Sunner, J.; Morales, A.; Kebarle, P. Anal. Chem. 1988, $60,98$.

35. Sunner, J.; Kulatunga, R.; Kebarle, P. Anal. Chem. 1986, 58, 1312 .

36. Todd, P. J.; Groenwold, G. S. Anal. Chem. 1986, 58, 895.

37. Kriger, M. S.; Cook, K. D.; Short, R. T.; Todd, P. J. Antal. Chem. 1992, 64, 3052.

38. Sunner, J., to be published.

39. Shiea, J; Sunner, J. Int. J. Mass Spectrom. Ion Processes 1991, $109,265$.

40. Junker, E.; Wirth, K. P.; Röllgen, F. W. I. Phys. 1989, C2, 53 .

41. McQuarrie, D. A. Statistical Thermodymamics; University Science Books: Mill Valley, CA, 1973; p 248.

42. Todd, P. J. J. Am. Soc. Mass Spectrom. 1991, 2, 33.

43. Shiea, J.; Sunner, J. Proceedings of the 38th Canference on Mass Spectrometry and Allied Topics; Tucson, $A Z, 1990 ; \mathrm{p} 166$.

44. Sunner, J.; Shiea, J. Proceedings of the 39th Conference on Mass Spectrontetry and Allied Topics; Nashville, TN, 1991; p 514.

45. Chait, B. T.; Kent, B. H. Science 1992, 257, 1885. 